(1)

CrossMark

\title{
The unknown pathophysiological relevance of right ventricular hypertrophy in pulmonary arterial hypertension
}

\author{
Frances S. de Man ${ }^{1}$, M. Louis Handoko ${ }^{2}$ and Anton Vonk-Noordegraaf ${ }^{1}$ \\ Affiliations: ${ }^{1}$ Amsterdam University Medical Center, Vrije universiteit Amsterdam, Dept of Pulmonary \\ Medicine, Amsterdam Cardiovascular Sciences, Amsterdam, The Netherlands. ${ }^{2}$ Amsterdam University \\ Medical Center, Vrije universiteit Amsterdam, Dept of Cardiology, Amsterdam Cardiovascular Sciences, \\ Amsterdam, The Netherlands.
}

Correspondence: Frances S. de Man, Amsterdam University Medical Center, Vrije universiteit Amsterdam, Dept of Pulmonary Medicine, Amsterdam Cardiovascular Sciences, De Boelelaan 1117, Amsterdam, The Netherlands. E-mail: fs.demandavumc.nl

@ERSpublications

A recent study clearly demonstrates that right ventricular (RV) hypertrophy is a prognostic marker of pulmonary arterial hypertension, triggering us to rethink the concept of RV hypertrophy http://ow.ly/ vD0e30nHvFm

Cite this article as: de Man FS, Handoko ML, Vonk-Noordegraaf A. The unknown pathophysiological relevance of right ventricular hypertrophy in pulmonary arterial hypertension. Eur Respir J 2019; 53: 1900255 [https://doi.org/10.1183/13993003.00255-2019].

The Greek king Sisyphus was punished by Zeus by being made to carry a huge boulder up a steep hill. Each time he thought he had reached the top, the boulder would roll down and Sisyphus would have to start all over again. Likewise, the right ventricle in patients with pulmonary arterial hypertension (PAH) is punished with every heart beat to cope with a 4-5-fold increase in load for the rest of its life [1]. As a consequence, the low-pressure, thin-walled, crescent-shaped ventricle has to transform into a ventricular shape that is able to pump against such an increased afterload [2]. Right ventricular (RV) hypertrophy is therefore observed in almost all PAH patients. RV hypertrophy is a necessary adaptation to lower RV wall tension, to increase the force generating capacity of the RV cardiomyocytes and to preserve RV-arterial coupling [3]. However, this adaptation might come at a price and becomes maladaptive in the end-stage of RV failure increasing stiffness [4] and oxygen consumption [5].

In the current issue of the European Respiratory Journal, SIMPSON et al. [6] have performed a thorough analysis to investigate the relationship between ventricular mass and survival in 64 incident PAH patients (42 connective tissue disease-associated PAH, 22 idiopathic PAH). During a median follow-up of 4.2 years, 30 deaths occurred ( $46 \%$ mortality), which demonstrates once again the devastating nature of this disease. Univariate analyses demonstrated that ventricular mass index (RV/left ventricular (LV) mass) and RV-end-diastolic mass index were closely associated with survival (both hazard ratio: 1.11 (95\% CI 1.03-1.19, $\mathrm{p}<0.01$ ). Also, after adjustment for demographic or haemodynamic parameters in multiple bivariate models, associations between markers of RV hypertrophy and survival continued to exist. Finally, significant correlations were observed between stroke volume/end-systolic volume (a measure of systolic RV function) and RV hypertrophy $(\mathrm{r}=-0.7 ; \mathrm{p}<0.01)$. Based on these data the authors suggest that RV hypertrophy may be 
maladaptive, and that mass-metrics may serve as early markers of maladaptive RV hypertrophy and remodelling.

This interesting finding raised the question of how an initial adaptation mechanism such as RV hypertrophy can be associated with increased mortality in end-stage PAH. However, it should be emphasised that these findings are strictly correlative and do not indicate that RV hypertrophy truly causes increased mortality in PAH patients. In addition, the study data should be interpreted with caution since the authors pooled the data of idiopathic $\mathrm{PAH}$ patients and patients with connective tissue disease-associated PAH. Whereas, earlier work demonstrated that the RV adaptation between these two groups of PAH differ significantly [7, 8].

Preclinical data is inconclusive as to whether hypertrophy should be targeted $[9,10]$. Several studies have demonstrated that preventing LV hypertrophy in mice with pressure overload due to transaortic banding does not result in reduced LV systolic dysfunction or increased mortality. Other studies have demonstrated negative effects of hypertrophy inhibition. In addition, SANO et al. [11] demonstrated the essential role of angiogenesis on hypertrophy development. Enhancing angiogenesis resulted in increased hypertrophy and preserved systolic function in a LV pressure overload model.

Only a small number of studies have investigated the effects of RV hypertrophy inhibition on RV adaptation to pressure overload. BOGAARD et al. [12] investigated the effect of an inhibitor of histone deacetylases (HDAC). HDAC inhibitors are known to reduce LV hypertrophy in murine models with transaortic constriction. Surprisingly, HDAC inhibitors did not reduce RV hypertrophy in a pulmonary artery banding model, but caused a switch from compensated RV hypertrophy to RV failure. Increased fibrosis and capillary rarefaction were the hallmarks of the observed maladaptive RV hypertrophy.

Another important regulator of hypertrophy is calcineurin [13]. Cyclosporine or FK506 are direct inhibitors of calcineurin and in such a way hypertrophy. FK506 is of special interest in PAH, as it is also a direct stimulator of BMPR2, and the clinical benefit of FK506 is currently being investigated [14]. Unfortunately, the direct effects of FK506 on RV hypertrophy and adaptation has not been thoroughly investigated yet [15].

There is clearly an unmet need for new studies in which the role of RV hypertrophy is further elucidated by either stimulating RV hypertrophy and enhancing angiogenesis or directly inhibiting RV hypertrophy at different stages of the disease. The study SIMPSON et al. [6] clearly demonstrates that RV hypertrophy is a prognostic marker of $\mathrm{PAH}$, triggering our minds to rethink the concept of RV hypertrophy. The results of this study urges us to explore the role of RV hypertrophy in PAH in more detail in the future.

Conflict of interest: None declared.

Support statement: This work was supported by Hartstichting (2018T059), Nederlandse Organisatie voor Wetenschappelijk Onderzoek (NWO-VICI: 918.16.610 and NWO-VIDI: 917.18.338) and the Netherlands CardioVascular Research Institute (CVON-2017-10 DOLPHIN-GENESIS and CVON-2018-29 PHAEDRA-IMPACT). Funding information for this article has been deposited with the Crossref Funder Registry.

\section{References}

1 Vonk Noordegraaf A, Chin KM, Haddad F, et al. Pathophysiology of the right ventricle and of the pulmonary circulation in pulmonary hypertension: an update. Eur Respir J 2019; 53: 1801900.

2 van der Bruggen CEE, Tedford RJ, Handoko ML, et al. RV pressure overload: from hypertrophy to failure. Cardiovasc Res 2017; 113: 1423-1432.

3 Rain S, Handoko ML, Trip P, et al. Right ventricular diastolic impairment in patients with pulmonary arterial hypertension. Circulation 2013; 128: 2016-2025.

4 Trip P, Rain S, Handoko ML, et al. Clinical relevance of right ventricular diastolic stiffness in pulmonary hypertension. Eur Respir J 2015; 45: 1603-1612.

5 Wong YY, Ruiter G, Lubberink M, et al. Right ventricular failure in idiopathic pulmonary arterial hypertension is associated with inefficient myocardial oxygen utilization. Circ Heart Fail 2011; 4: 700-706.

6 Simpson CE, Damico RL, Kolb TM, et al. Ventricular mass as a prognostic imaging biomarker in incident PAH. Eur Respir J 2019; 53: 1802067.

7 Overbeek MJ, Lankhaar JW, Westerhof N, et al. Right ventricular contractility in systemic sclerosis-associated and idiopathic pulmonary arterial hypertension. Eur Respir J 2008; 31: 1160-1166.

8 Hsu S, Kokkonen-Simon KM, Kirk JA, et al. Right ventricular myofilament functional differences in humans with systemic sclerosis-associated versus idiopathic pulmonary arterial hypertension. Circulation 2018; 137: 2360-2370.

9 Schiattarella GG, Hill TM, Hill JA. Is load-induced ventricular hypertrophy ever compensatory? Circulation 2017; 136: $1273-1275$.

10 Carabello BA. Is cardiac hypertrophy good or bad? The answer, of course, is yes. JACC Cardiovasc Imaging 2014; 7: 1081-1083.

11 Sano M, Minamino T, Toko H, et al. p53-induced inhibition of Hif-1 causes cardiac dysfunction during pressure overload. Nature 2007; 446: 444-448. 
12 Bogaard HJ, Mizuno S, Hussaini AA, et al. Suppression of histone deacetylases worsens right ventricular dysfunction after pulmonary artery banding in rats. Am J Respir Crit Care Med 2011; 183: 1402-1410.

13 Schiattarella GG, Hill JA. Inhibition of hypertrophy is a good therapeutic strategy in ventricular pressure overload. Circulation 2015; 131: 1435-1447.

14 Spiekerkoetter E, Sung YK, Sudheendra D, et al. Randomised placebo-controlled safety and tolerability trial of FK506 (tacrolimus) for pulmonary arterial hypertension. Eur Respir J 2017; 50: 1602449.

15 Spiekerkoetter E, Tian X, Cai J, et al. FK506 activates BMPR2, rescues endothelial dysfunction, and reverses pulmonary hypertension. J Clin Invest 2013; 123: 3600-3613. 